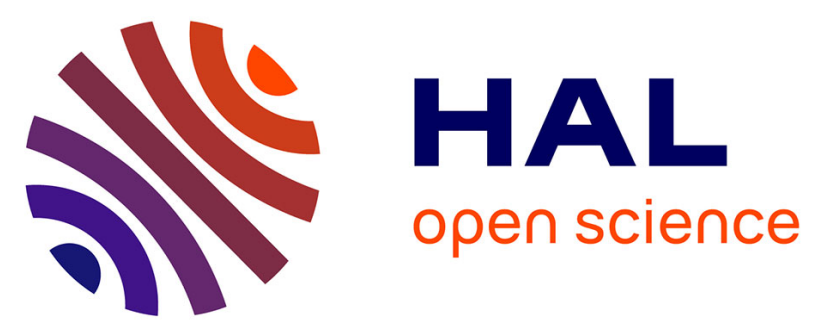

\title{
Long-memory dynamics in a SETAR model - Applications to stock markets
}

Gilles Dufrénot, Dominique Guegan, Anne Peguin-Feissolle

\section{To cite this version:}

Gilles Dufrénot, Dominique Guegan, Anne Peguin-Feissolle. Long-memory dynamics in a SETAR model - Applications to stock markets. Journal of International Financial Markets, Institutions and Money, 2005, 15, pp.391 - 406. 10.1016/j.intfin.2004.09.001 . halshs-00179339

\section{HAL Id: halshs-00179339 \\ https://shs.hal.science/halshs-00179339}

Submitted on 15 Oct 2007

HAL is a multi-disciplinary open access archive for the deposit and dissemination of scientific research documents, whether they are published or not. The documents may come from teaching and research institutions in France or abroad, or from public or private research centers.
L'archive ouverte pluridisciplinaire HAL, est destinée au dépôt et à la diffusion de documents scientifiques de niveau recherche, publiés ou non, émanant des établissements d'enseignement et de recherche français ou étrangers, des laboratoires publics ou privés. 


\title{
Long-memory dynamics in a SETAR model - Applications to stock markets ${ }^{\ddagger}$
}

\author{
Gilles DUFRENOT ${ }^{\ddagger}$ Dominique GUEGAN ${ }^{\S}$ \\ Anne PEGUIN-FEISSOLLEף
}

February 20, 2004

\begin{abstract}
This paper presents a 2-regime SETAR model with a long-memory process in the first regime and a short-memory process in the second regime. We briefly introduce the properties of this model and methods for locating the threshold parameter are proposed. Such a process is applied to stock indices and individual asset prices. A comparison with simple FARIMA models is made using some forecastibility criteria.
\end{abstract}

Keywords: SETAR - Long-memory - Stock indices - Forecasting.

JEL classification: C32, C51, G12

*Corresponding author: Anne PEGUIN-FEISSOLLE, GREQAM, Centre de la Vieille Charité, 2 rue de la Charité, 13002 Marseille, France, tel: +33.4.91.14.07.35, fax: +33.4.91.90.02.27, Email: peguin@ehess.univ-mrs.fr

${ }^{\dagger}$ The authors gratefully acknowledge the comments from the participants to the International Conference on Forecasting Financial Markets, in Paris, June 2003, and to the Workshop "Models with Breaks in Economics and Finance: Recent Developments", in Paris, November 2003. They also thank the Conseil Régional of PACA for its financial support and Aldric Pouron for providing them with the data.

‡ERUDITE, Université Paris 12 and GREQAM-CNRS, 2 rue de la Vieille Charité, 13002 Marseille, France, Email: lopaduf@aol.com

${ }^{\S}$ Ecole Normale Supérieure de Cachan, MORA-IDHE, UMR-CNRS, and Senior Academic Fellow de l'IEF, 61 avenue du Président Wilson, 94235, Cachan, Cédex, France, Email:guegan@ecogest.ens-cachan.fr

`GREQAM-CNRS, Centre de la Vieille Charité, 2 rue de la Vieille Charité, 13002 Marseille, France, Email: peguin@ehess.univ-mrs.fr 


\section{Introduction}

In economics and finance, multiple regimes modeling becomes more and more important in order to take into account phenomena characterized for instance by recession or expansion periods, or high or low volatility periods. Consequently, a variety of models has been proposed in the literature to account for this behavior, among which stochastic volatility models, nonlinear GARCH models, threshold autoregressive models (TAR), Markov switching models, multifactor GARCH models, etc.. Here, we focus our attention on the TAR models that represent one of the most prominent classes of regime-switching models. They have been initially introduced by Tong and Lim (1980) and discussed in Tong (1990)). They assume that the regimes are determined by a structural indicator relied on the observed process; when this threshold variable is the time series itself, as we consider in this paper, the model is called a Self-Exciting TAR (SETAR) model.

Moreover, each regime in the well-known TAR model is represented by a short memory relation (generally an autoregressive equation). But it is worthwhile noting here that economic or financial data can present sometimes a long memory behavior in one or both regimes and it could be interesting to consider models with the possibility of long memory behavior, at least in one regime. Accordingly, in this paper, we propose to describe the regimes using a SETAR model with one regime characterized by a long-memory dynamics and the other by weak dependence; we thus consider a process $\left(X_{t}\right)_{t}$ that satisfies the following scheme: $\forall t$,

$$
\left\{\begin{array}{cl}
(1-B)^{d} X_{t}=\varepsilon_{t}^{(1)}, & \text { if } X_{t-1} \leq c: \text { regime } 1 \\
X_{t}=\varepsilon_{t}^{(2)}, & \text { if } X_{t-1}>c: \text { regime } 2
\end{array}\right.
$$

or

$$
\left\{\begin{array}{ccc}
X_{t}=\varepsilon_{t}^{(1)}, & \text { if } X_{t-1} \leq c: & \text { regime } 1 \\
(1-B)^{d} X_{t}=\varepsilon_{t}^{(2)}, & \text { if } X_{t-1}>c: & \text { regime } 2,
\end{array}\right.
$$

where $d \in[0,1 / 2]$ is a fractional difference parameter, $c$ is the threshold parameter, $\varepsilon_{t}^{(i)}, i=1,2$ are strong white noises with finite variances and $B$ is the backward shift operator. So, we introduce a persistent dynamics in at least one regime.

Therefore, this paper belongs to a literature exploring simultaneously two key properties of the financial time series, namely the long-memory and nonlinear properties. Empirical works have been done along two lines of research. 
- A first line of papers enhances a skeptic view. The long-memory property uncovered in the financial data would reflect strong biases in presence of neglected nonlinearities. Authors sharing this view argue that neglected switching dynamics or regime shifts imply spurious longmemory structures. Papers that highlight such a confusion include Hidalgo and Robinson (1996), Lobato and Savin (1997), Bos, Franses and Ooms (1999), Granger and Hyung (1999), Diebold and Inoue (2001) and Breidt and Hsu (2002). Diebold and Inoue (2001) provide some Monte Carlo results suggesting that spurious long-memory characterize many nonlinear models currently applied to economic data, among which the Markov switching models. A consequence of this pessimistic view is that nonlinearity and long-memory should be held jointly if one wants to be able to evaluate their relative importance. These papers are based on Allan (1966)'s long memory definition. Here, we adopt the long memory approach defined by Granger (1980).

- Accordingly, a second strand of the literature has recently proposed "nonlinear long-memory" models. For instance, some authors provide a joint evidence of mean reversion over long horizons and nonlinear dynamics on exchange rate markets, by generalizing to the nonlinear framework the Beveridge Nelson decomposition (see, Clarida and Taylor (2001), Sarno and Taylor (2001)). Others propose new classes of long-memory models. For instance, Franses and Paap (2002), Franses, Van der Leij and Paap (2002) introduce CLEAR and Switching CLEAR processes, which show autocorrelation at high lags with an ACF that decays at a faster rate in the beginning in comparison to the ACF of an FARIMA model. Guégan (2000, 2003) introduces the GIGARCH model which allies at the same time long-memory, seasonality and heteroscedasticity effects, with application to inflation rates. Fractionally integrated threshold models have also been proposed, that offers another potential application to financial data (see van Dick, Franses and Paap (2002)).

This paper is mainly oriented on applications of models (1) and (2); we show that these models provide more accurate forecasts than standard long memory models.

The plan of the paper is as follows. Section 2 briefly presents the memory properties of the model including a long-memory regime and proposes some methods for the location of the unknown parameter $c$. Section 3 contains some empirical applications to financial data. Section 4 concludes the paper. 


\section{Properties of the model and estimation}

We refer to the "long-memory" definition in Granger (1980)'s sense: a long memory behavior can be represented by an autocorrelation function decreasing slowly towards 0 . More precisely, we say that a stationary process $\left(X_{t}\right)_{t}$, whose autocovariance function is $\gamma_{X}$, is long memory if, $\forall t$ and $\forall \tau$,

$$
\gamma_{X}(\tau) \sim C(d) \tau^{2 d-1} \quad \text { as } \tau \rightarrow \infty
$$

where $0<d<1 / 2$ and $C(d)$ is a constant which depends only on $d$. Under the assumption that $\varepsilon_{t}^{(i)}, i=1,2$, are strong white noises with finite variances, model (1) is both locally (in each regime) and globally stationary and invertible. Its autocorrelation function, $\gamma_{X}(\tau)$, can be written as:

$$
\gamma_{X}(\tau)=C(\tau, d) N_{1}(c)+\gamma_{\varepsilon}(0) N_{2}(c),
$$

where

$$
C(\tau, d)=\frac{\Gamma(1-2 d) \Gamma(\tau+d)}{\Gamma(d) \Gamma(1-d) \Gamma(\tau+1-d)}
$$

$N_{1}(c)$ and $N_{2}(c)$ are the percentages of observations, respectively in regime 1 and in regime 2 and are not pre-defined, but depends upon the threshold c. $\gamma_{\varepsilon}(0)$ is the variance of the noise $\left(\varepsilon_{t}^{(2)}\right)_{t}$. The theoretical autocorrelation function is thus a mixture of the autocorrelation of the long-memory model in regime 1 and of the variance of the white noise model in regime 2 . It can be shown, via simulations, that $\gamma_{X}(\tau)$ exhibits a variety of decay rates (fast to very slow) according to the values of $c$ (see Dufrénot, Guégan and Péguin-Feissolle (2003)).

Even in a simple formulation such as ours, the estimation of the model parameters $(d, c)$ is substantially more difficult than in the cases of FARIMA models or standard SETAR models. Ideally, one would like to apply here methods based on maximum likelihood approach (the Whittle estimator), but this is not feasible because the model is piecewise linear. Further, it is not possible to apply the variety of methods suggested for standard SETAR models because of the presence of the fractional parameter in one regime. The approach we adopt here is sequential as follows:

- One estimates the value of the threshold parameter $c$.

- The observations are separated in two sub-groups according to the estimated value of $c$ and one deduces $N_{1}(c), N_{2}(c)$. 
- Classical methods are applied to each sub-group to obtain an estimation of the fractional parameter (GPH, non-parametric methods, Whittle). If the model (1) (or (2)) is adequate to describe the data, then one must find a significant parameter $d$ for one sub-group and a non significant parameter for the other sub-group.

The crucial point concerns the first step where the parameter $c$ has to be estimated. We first construct the time series $\left(\widetilde{X}_{t}\right)_{t}$ of arranged observations according to the decreasing values of $X_{t-1}$ and then proceed as follows:

1. One considers a set of $s_{1}$ initial observations of $\left(\widetilde{X}_{t}\right)_{t}$ and estimate the long-memory parameter and the corresponding $t-$ ratio: $t_{s_{1}}$.

2. The vector $\left(\widetilde{X}_{t}\right)_{t}$ is then incremented in such a way to contain $s_{2}, s_{3}$, $\ldots, s_{n}$ observations; new long-memory parameters and their $t$-ratios are computed: $t_{s_{2}}, t_{s_{3}}, \ldots, t_{s_{n}}$ (in the applications, we will add just one observation: $s_{j}=s_{j-1}+1$ for all $j$ ).

3. Consider the set of estimated t-ratios $\left\{t_{s_{1}}, t_{s_{2}}, t_{s_{3}}, \ldots, t_{s_{n}}\right\}$. One tests for the presence of a structural break $\widetilde{t}$ in the view of finding a sequence of t-ratios such that, for instance, for $t_{s_{k}} \leq \widetilde{t}$, the estimated long-memory parameters are not statistically significant, while, for $t_{s_{k}}>\widetilde{t}$, they are significant.

A simple way to do this is to use a standard Chow test. The series of tratios is regressed on a linear time trend, using incremented dummy variables: for $k=1,2, \ldots, n$

$$
t_{s_{k_{t}}}=\left(\alpha+\beta D_{t}\right)+\left(\gamma+\delta D_{t}\right) t+u_{t},
$$

where $u_{t}$ is a strong white noise and

$$
D_{t}=\left\{\begin{array}{l}
1, \quad \text { if } t \leq \widetilde{t} \\
0, \text { otherwise. }
\end{array}\right.
$$

We test the null hypothesis $H_{0}: \beta=\delta=0$ against the alternative $\beta \neq 0$ or $\gamma \neq 0$. The constant term is omitted if we want to test changes only in the slope. The test is implemented by considering different values of $\widetilde{t}$ and finally one retains the value yielding the lowest p-value. Instead of using the Chow test, one can also compute the sum of squares residuals corresponding to the equation and select the $t$-ratio (and thus the threshold value) yielding the lowest sum. 


\section{Empirical applications}

We consider different asset prices and five stock indices $p_{t}, t=1, \ldots, T$. The data are daily and run from 1997 to 2003. The names of the companies and indices are listed in table 1.

We consider the power returns

$$
X_{t}=R_{t}^{\delta}
$$

with

$$
R_{t}=\log \left(p_{t}\right)-\log \left(p_{t-1}\right)
$$

and $\delta=2$ or 4 , i.e. we are interested in modeling the processes $\left(R_{t}^{2}\right)_{t}$ and $\left(R_{t}^{4}\right)_{t}$. When $\delta=2$, the squared returns are taken as proxies of the instantaneous and empirical volatility, while, when $\delta=4$, the power returns capture the dynamics of the kurtosis.

\subsection{Case 1: the process $\left(R_{t}^{2}\right)_{t}$}

Table 2 shows our estimation results. The data successfully detect the presence of two distinct regimes in the volatility of three stock indices. To save place, we report the results for which the change point in the t-ratios is obtained using the Chow-based method (the approach based on the minimum sum of squares yields similar results). Further, we report the estimations of the fractional parameters based on the GPH method: $\widehat{d}$ corresponds to a simple FARIMA model on the whole series, and $\widehat{d}_{1}$ and $\widehat{d}_{2}$ to the fractional parameters of both regimes in model (1) or (2); $\widehat{c}$ is the value of the threshold parameter, $N_{1}$ and $N_{2}$ are the number of points in the regimes and the last row gives the corresponding model (1) or (2).

Several comments are in order.

- Firstly, comparing the t-ratios of the fractional parameter in the two regimes, we find evidence that both the models (1) and (2) are able to capture the state-dependent dynamics of the volatility. For instance, in the case of ST-GOBAIN, the low volatility regime is characterized by some predictable slow dynamic adjusting behavior, i.e. correspond to model (1), whereas for TOTAL the volatility is highly persistent in the regime of large past volatility (model (2)). These results suggest that the two facets of volatility clustering - low volatility clustering and high volatility clustering- can occur separately if one does not force the volatility data-generating process to be only one regime (as is the case of GARCH models). 
- A second point is that, for these two indices, we approximately have the same number of observations within each regimes. The implications for market analysts is that strategies based on technical analysis (charts, market timing, dynamic asset allocation) and those based on investment strategies (for instance, portfolio insurance) are not mutually exclusive. The first one can be used when a persistent volatility generating process is detected and the second one during the periods of unpredictable volatilities.

- A third interesting point concerns the difference between an aggregate stock index like FTSE and disaggregated firms' stock indices. Although we find a two-regime model for the FTSE index, it is seen that the number of points in the white noise regime is only approximately $10 \%$ of the sample. We can observe that our "on/off" persistent volatility model captures the influence of market microstructures that would not be found when individual assets are aggregated.

Finally, it is interesting to enquire as to whether a model where the persistence of the volatility is allowed in one regime only produces forecasts that are superior to a one-regime long-memory model. Table 3 reports some results of predictive accuracy based on Diebold and Mariano (1995)'s test statistics. The number in bold correspond to cases where the SETAR model yields forecasts that are statistically different from those obtained with a simple FARIMA model. The number $\mathrm{n}$ in the table indicates the percentages of points for which the residuals of our model are inferior to those of the one-regime FARIMA model. As is seen, the numbers are higher than 50\%, which suggests that our model offers an alternative competing framework to describe the persistence of the volatility.

\subsection{Case 2: the process $\left(R_{t}^{4}\right)_{t}$}

It seems worthwhile to look at some possible extensions of the preceding results: we investigated thus the case corresponding to $\delta=4$ in (2). Considering the power 4 of a process $\left(R_{t}\right)_{t}$ is a way to measure the kurtosis of its underlying distribution. The behavior of the kurtosis is important in the Value at Risk analysis (VaR). The latter can be viewed as a measure of the maximum loss of a portfolio over a predetermined horizon:

$$
\operatorname{VaR}=-C \cdot V_{h}
$$

where $C$ is the current market value of a portfolio and $h$ the holding period. $V_{h}$ is the cut-off value which is exceeded by $h$-period return with probability 
$1-p:$

$$
V_{h}=F_{t, h}^{-1}(p),
$$

where $F_{t, h}($.$) is the probability distribution of the h$-period return $R_{t, h}$. Generally $F_{t, h}($.$) is analytically intractable, especially when h$ is large (see Brummelhuis and Guégan (2004)). In this context, the knowledge of the time varying behavior of moments like the fourth-order moments is very useful to get a better accuracy for the parameters of the distribution function $F_{t, h}($.$) ;$ this permits to compute the $V_{h}$ and in turn the VaR. Models based on the Kurtosis improved the predictions of the VaR, especially for aggregate returns (see Wong and So (2003)).

Table 4 contains the estimation results. We successfully detect the presence of two distinct regimes for eight stock indices; a peculiar case is ST GOBAIN where no long memory is found in a simple FARIMA model on the whole period but where we find evidence that a 2-regime model is able to capture the state-dependent dynamics of the series.

Further, the data successfully detect the presence of two distinct regimes in the series $R_{t}^{4}$ of five asset indices and three aggregate stock indices. More precisely, we have

- for three series (CARREFOUR, FTSE and SBF), the adequate model is the model (1), i.e. the low past $R_{t}^{4}$ regime is characterized by some long memory dynamics;

- for the other five series (FRANCETEL, BNP, LOREAL, ST GOBAIN and CAC40), the model (2) is detected, meaning that the process $R_{t}^{4}$ is highly persistent for large past $R_{t}^{4}$.

Now, we use the estimated models to make predictions. Table 5 shows that these predictions are higher with the model (1) or (2) than with a simple FARIMA model: the number $n$ in the table, i.e. the percentages of points for which the residuals of our model are inferior to those of the one-regime FARIMA model, is very high, between $54 \%$ and $75 \%$ (except in the case of ST GOBAIN), suggesting that our model offers an interesting competitive modeling approach to describe the persistence of the process $R_{t}^{4}$.

Moreover, we observe that these predictions increase with the lag $h$ (not shown here but available upon request to authors). The behavior of the kurtosis will influence the computation of the VaR introduced in (8), particularly when $h$ increases. Indeed, excess kurtosis measures the tail thickness of the distribution and a positive excess of kurtosis indicates a leptokurtic distribution. 
Thus, in the context of the risk management theory it seems important to consider the use of model (1) or (2) which permits to separate the data governed by a short memory process and those governed by a long memory process. The knowledge of the time varying behavior of moments like the fourth order moments could be very useful to get a better accuracy for the parameters of the distribution function $F_{t, h}($.$) which permits to compute the$ $V_{h}$ and thus the $V a R$. This approach seems particularly appropriate for a portfolio which corresponds to an aggregation of returns. Wong and So (2003) showed that they improve the predictions of the $\operatorname{VaR}$ using models on the skewness and kurtosis based on an heteroscedastic modeling.

\section{Conclusion}

This paper proposes a new model to investigate the long-memory dynamics of time series that contains a mixture of long-memory and white noise structures in a 2-regime SETAR. To pick up the part of the long-memory in the data, we give some methods in order to locate the threshold parameter. Our empirical results suggest that our model offers an interesting alternative competing framework to describe the persistent dynamics in modeling the processes $\left(R_{t}^{2}\right)_{t}$ and $\left(R_{t}^{4}\right)_{t}$.

Some further research topics are the following. Firstly, one can conjecture that the model suggested here can be extended to the case where regime 1 is described by an FARIMA model and regime 2 by a stationary ARMA model or by a mixing process. Such a question is interesting since an FARIMA process is not mixing (see Guégan and Ladoucette (2001)). Secondly, the model can be applied to other time series than the transformation of returns. Variables based on technical trading rules can be suggested (for instance, a variable constructed from a short-run moving average and a long-run moving average) in order to capture asymmetric dynamics in the memory structure. Thirdly, the SETAR model with long-memory behavior can be studied under the assumption of heteroskedastic errors in order to incorporate the influence of volatility of the long-memory structure. 


\section{References}

[1] Allan, D.W., 1966, Statistics of atomic frequency standards, Proceedings IEEE 54, 221-230.

[2] Bos, C., P. H. Franses and M. Ooms, 1999, Re-analyzing inflation rates: evidence of long-memory and level shifts, Empirical Economics 24, 427449.

[3] Breidt, F.J. and N.J. Hsu, 2002, A class of nearly long memory processes, International Journal of Forecasting 18, 265-281.

[4] Brummelhuis, R. and D. Guégan, 2004, Multi-period conditional distribution functions for heteroscedastic models with applications to VaR, forthcoming in Journal of Applied Probability.

[5] Clarida, R. H. and M. P. Taylor, 2001, Nonlinear permanent-temporary decomposition, with applications in macroeconomics and finance, Working Paper, University of Warwick.

[6] Diebold, F. X. and A. Inoue, 2001, Long-memory and regime switching, Journal of Econometrics, 105, 131-159.

[7] Diebold, F. X. and R. Mariano, 1995, Comparing predictive accuracy, Journal of Business and Economic Statistics, 253-263.

[8] Dufrénot, G., D. Guégan and A. Péguin-Feissolle, 2003, A SETAR model with long-memory property, International Conference on Forecasting Financial Markets, Paris, June.

[9] Franses, P. H. and R. Paap, 2002, Censored latent effects autoregression, with an application to US unemployment, Journal of Applied Econometrics, 17, 347-366.

[10] Franses, P. H., M. van Der Leij and R Paap, 2002, Modelling and forecasting level shifts in absolute returns, Journal of Applied Econometrics, 17, 601-616.

[11] Granger, C.W.J., 1980, Long memory relationships and the aggregation of dynamic models, Journal of Econometrics, 14, 227 - 238.

[12] Granger, C. W. J. and N. Hyung, 1999, Occasional breaks and longmemory, Discussion paper 99-14, University of California, San Diego. 
[13] Guégan, D., 2000, A new model: the $k$-Factor GIGARCH Process, Journal of Signal Processing, 4, 265 - 271.

[14] Guégan, D., 2003, A Prospective study of the $k$-Factor Gegenbauer Processes with heteroscedastic errors and an application to inflation rates, Finance India, XVII, 165-197.

[15] Guégan D. and S. Ladoucette, 2001, Non mixing properties of long memory processes, C.R.A.S. 333, Ser. I, 373-376.

[16] Hidalgo, J. and P. M. Robinson, 1996, Testing for structural change in a long-memory environment, Journal of Econometrics, 70, 159-174.

[17] Lobato, I. N. and N. E. Savin, 1997, Real and spurious long-memory properties of stock-market data, Journal of Business and Economic Statistics, 16, 261-283.

[18] Sarno, L. and M. Taylor, 2001, Real exchange rate dynamics in transition economies: a nonlinear analysis, Studies in Nonlinear Dynamics and Econometrics, 5, 153-177.

[19] Tong, H., 1990, Non-linear time series; a dynamical systems approach (Oxford University Press).

[20] Tong, H. and K.S. Lim, 1980, Threshold autoregressions, limit cycles, and data, Journal of the Royal Statistical Society B, 42, 245-292 with discussions.

[21] van Dijk, D., P. H. Franses and R. Paap, 2002, A nonlinear long-memory model with an application to US unemployment, Journal of Econometrics, 110, 135-165.

[22] Wong, C.M. and M.P. So, 2003, On conditional moments of GARCH models with applications to multiple period value at risk estimation, Statistica Sinica 13, 1015 - 1044. 
Table 1. Names of companies and indices

\begin{tabular}{lc}
\hline Companies or indices & period \\
\hline \hline France Télécom & $20 / 10 / 97-23 / 04 / 03$ \\
Bnp & $02 / 01 / 98-23 / 04 / 03$ \\
Carrefour & $02 / 01 / 98-23 / 04 / 03$ \\
Loreal & $02 / 01 / 98-23 / 04 / 03$ \\
St Gobain & $02 / 01 / 98-23 / 04 / 03$ \\
Total FinaElf & $02 / 01 / 98-23 / 04 / 03$ \\
Ftse & $02 / 01 / 98-23 / 04 / 03$ \\
Cac40 & $01 / 05 / 98-23 / 04 / 03$ \\
Sbf & $02 / 01 / 98-23 / 04 / 03$ \\
\hline
\end{tabular}


Table 2. Estimation of parameters for $R_{t}^{2}$

\begin{tabular}{cccc}
\hline & ST GOBAIN & TOTAL & FTSE \\
\hline \hline$\widehat{d}$ & 0.0919 & 0.1066 & 0.3165 \\
t-ratio & $(3.09)$ & $(2.51)$ & $(8.90)$ \\
$\widehat{c}$ & 0.0001 & 0.0002 & 0.0006 \\
$N_{1}$ & 606 & 740 & 1236 \\
$\widehat{d_{1}}$ & 0.0904 & 0.0560 & 0.2907 \\
t-ratio & $(3.29)$ & $(1.37)$ & $(6.90)$ \\
$N_{2}$ & 734 & 600 & 102 \\
$\widehat{d}_{2}$ & 0.1064 & 0.1615 & 0.1835 \\
t-ratio & $(1.93)$ & $(3.06)$ & $(1.49)$ \\
model & $(1)$ & $(2)$ & $(1)$ \\
\hline
\end{tabular}

Note: The change point in the t-ratios was obtained using the method based on the Chow test. All the estimations are made with GPH method. $\widehat{d}_{1}$ and $\widehat{d}_{2}$ refer to the estimated fractional parameter and $N_{1}$ and $N_{2}$ the number of observations, respectively in regime 1 and regime 2. $\widehat{d}$ is the estimated fractional parameter on the whole series. The t-ratio must be compared 1.96 (corresponding to the critical value at the $5 \%$ level of significance). A non-significant parameter indicates that the volatility is driven by a white noise process (and is thus unpredictable). Conversely, a significant parameter means that the volatility exhibit a long-memory dynamics therefore yielding to a high predictability. The last row shows the corresponding adequate model (1) or (2). 
Table 3. Predictive accuracy testing for $R_{t}^{2}$ (p-values)

\begin{tabular}{lrrrrrrc}
\hline & \multicolumn{1}{c}{ AS } & \multicolumn{1}{c}{ SI } & \multicolumn{1}{c}{ WI } & \multicolumn{1}{c}{ NB } & MGN & MR & $\mathrm{n}$ \\
\hline \hline ST GOBAIN & $\mathbf{0 . 0 6 7}$ & $\mathbf{0 . 0 0 0}$ & 0.403 & 0.155 & 0.310 & 0.113 & $55.19 \%$ \\
TOTAL & $\mathbf{0 . 0 0 0}$ & 0.396 & 0.232 & $\mathbf{0 . 0 4 1}$ & $\mathbf{0 . 0 8 2}$ & 0.113 & $51.16 \%$ \\
FTSE & 0.488 & $\mathbf{0 . 0 0 0}$ & $\mathbf{0 . 0 0 9}$ & 0.199 & 0.391 & 0.250 & $55.57 \%$ \\
\hline
\end{tabular}

Note: The different columns are: AS: Asymptotic test, SI: Sign test, WI: Wilcoxon's test, NB: Naive benchmark test, MGN: Morgan-Granger-Newbold's test, MR: MeeseRogoff's test, n: number of times in percent where the residuals coming from the TAR model with a long memory regime are smaller than the residuals coming from a standard long memory model (when $\mathrm{n}_{\llcorner} 50 \%$, it means that the TAR model seems the best). The null hypothesis is the hypothesis of equal accuracy of different predictive methods. The loss function is quadratic. The test statistics follows asymptotically different distributions: $N(0,1)$ for the asymptotic test, the sign test, the Wilcoxon's test, the Meese-Rogoff's test, $F\left(T_{0}, T_{0}\right)$ for the Naive benchmark test and a $t_{T_{0}-1}$ for the Morgan-Granger-Newbold's test (where $T_{0}$ is the number of predicted observations, i.e. $T_{0}=20$ ). The Meese-Rogoff test statistic is computed with the Diebold-Rudebusch covariance matrix estimator. The truncation lag is 10 for the asymptotic test and is given by the integer part of $T_{0}^{4 / 5}$ for the Meese-Rogoff's test. 
Table 4. Estimation of parameters for $R_{t}^{4}$

\begin{tabular}{ccccc}
\hline & FRANCETEL & BNP & CARREFOUR & LOREAL \\
\hline \hline$\widehat{d}$ & 0.1807 & 0.1895 & 0.1205 & 0.2262 \\
t-ratio & $(4.85)$ & $(5.53)$ & $(3.34)$ & $(6.39)$ \\
$\widehat{c}$ & $4 \times 10^{-6}$ & $4 \times 10^{-10}$ & $5 \times 10^{-8}$ & $2 \times 10^{-7}$ \\
$N_{1}$ & 1132 & 257 & 723 & 940 \\
$\widehat{d}_{1}$ & 0.0148 & 0.0176 & 0.1229 & 0.0615 \\
t-ratio & $(0.61)$ & $(0.22)$ & $(2.29)$ & $(1.42)$ \\
$N_{2}$ & 258 & 1082 & 616 & 400 \\
$\widehat{d}_{2}$ & 0.0912 & 0.1720 & 0.0731 & 0.3249 \\
t-ratio & $(3.22)$ & $(4.36)$ & $(1.31)$ & $(5.21)$ \\
model & $(2)$ & $(2)$ & $(1)$ & $(2)$ \\
\hline
\end{tabular}

\begin{tabular}{ccccc}
\hline & ST GOBAIN & FTSE & CAC40 & SBF \\
\hline \hline$\widehat{d}$ & 0.0037 & 0.2820 & 0.1594 & 0.1427 \\
t-ratio & $(0.77)$ & $(8.09)$ & $(4.35)$ & $(3.89)$ \\
$\widehat{c}$ & $2 \times 10^{-8}$ & $2 \times 10^{-7}$ & $2 \times 10^{-9}$ & $3 \times 10^{-9}$ \\
$N_{1}$ & 615 & 1200 & 489 & 550 \\
$\widehat{d}_{1}$ & 0.0077 & 0.2514 & 0.1079 & 0.2093 \\
t-ratio & $(1.87)$ & $(7.10)$ & $(1.61)$ & $(4.55)$ \\
$N_{2}$ & 725 & 138 & 850 & 790 \\
$\widehat{d}_{2}$ & 0.2691 & 0.1622 & 0.1453 & -0.0024 \\
t-ratio & $(6.90)$ & $(1.95)$ & $(2.92)$ & $(-0.05)$ \\
model & $(2)$ & $(1)$ & $(2)$ & $(1)$ \\
\hline
\end{tabular}

Note: The change point in the t-ratios was obtained using the method based on the Chow test. All the estimations are made with GPH method. $\widehat{d}_{1}$ and $\widehat{d}_{2}$ refer to the estimated fractional parameter and $N_{1}$ and $N_{2}$ the number of observations, respectively in regime 1 and regime 2. $\widehat{d}$ is the estimated fractional parameter on the whole series. The t-ratio must be compared 1.96 (corresponding to the critical value at the $5 \%$ level of significance). A non-significant parameter indicates that the volatility is driven by a white noise process (and is thus unpredictable). Conversely, a significant parameter means that the volatility exhibit a long-memory dynamics therefore yielding to a high predictability. The last row shows the corresponding adequate model (1) or (2). 
Table 5. Predictive accuracy testing for $R_{t}^{4}$ (p-values)

\begin{tabular}{lccccccc}
\hline & AS & SI & WI & NB & MGN & MR & $\mathrm{n}$ \\
\hline \hline FRANCETEL & 0.918 & $\mathbf{0 . 0 0 0}$ & $\mathbf{0 . 0 0 0}$ & 0.518 & 0.962 & 0.851 & $74.59 \%$ \\
BNP & 0.983 & $\mathbf{0 . 0 0 1}$ & $\mathbf{0 . 0 0 0}$ & 0.522 & 0.954 & 0.308 & $54.48 \%$ \\
CARREFOUR & 0.196 & $\mathbf{0 . 0 0 0}$ & $\mathbf{0 . 0 0 0}$ & 0.232 & 0.463 & $\mathbf{0 . 0 3 2}$ & $74.14 \%$ \\
LOREAL & 0.331 & $\mathbf{0 . 0 0 0}$ & $\mathbf{0 . 0 0 0}$ & 0.102 & 0.201 & $\mathbf{0 . 0 0 0}$ & $71.02 \%$ \\
ST GOBAIN & 0.801 & $\mathbf{0 . 0 0 0}$ & $\mathbf{0 . 0 0 0}$ & 0.496 & 0.992 & 0.817 & $41.90 \%$ \\
FTSE & 0.929 & $\mathbf{0 . 0 0 0}$ & $\mathbf{0 . 0 0 0}$ & 0.456 & 0.911 & 0.833 & $59.99 \%$ \\
CAC40 & 0.470 & $\mathbf{0 . 0 0 0}$ & $\mathbf{0 . 0 0 0}$ & 0.349 & 0.699 & 0.175 & $61.81 \%$ \\
SBF & $\mathbf{0 . 0 0 0}$ & $\mathbf{0 . 0 0 0}$ & $\mathbf{0 . 0 0 0}$ & 0.290 & 0.579 & 0.238 & $66.09 \%$ \\
\hline
\end{tabular}

Note: The different columns are: AS: Asymptotic test, SI: Sign test, WI: Wilcoxon's test, NB: Naive benchmark test, MGN: Morgan-Granger-Newbold's test, MR: MeeseRogoff's test, n: number of times in percent where the residuals coming from the TAR model with a long memory regime are smaller than the residuals coming from a standard long memory model (when $\mathrm{n}_{i} 50 \%$, it means that the TAR model seems the best). The null hypothesis is the hypothesis of equal accuracy of different predictive methods. The loss function is quadratic. The test statistics follows asymptotically different distributions: $N(0,1)$ for the asymptotic test, the sign test, the Wilcoxon's test, the Meese-Rogoff's test, $F\left(T_{0}, T_{0}\right)$ for the Naive benchmark test and a $t_{T_{0}-1}$ for the Morgan-Granger-Newbold's test (where $T_{0}$ is the number of predicted observations, i.e. $T_{0}=20$ ). The Meese-Rogoff test statistic is computed with the Diebold-Rudebusch covariance matrix estimator. The truncation lag is 10 for the asymptotic test and is given by the integer part of $T_{0}^{4 / 5}$ for the Meese-Rogoff's test. 